\title{
NON-STATIONARY EFFECTS IN HYPERSONIC NONUNIFORM DUSTY-GAS FLOW PAST A BLUNT BODY*
}

\author{
A.N. Osiptsov A.V. Rozin \\ (Institute of Mechanics, Lomonosov Moscow State University, 119899 Moscow, Russia) \\ Wang Boyi (王柏雾) \\ (Institute of Mechanics, Chinese Academy of Sciences, Beijing 100080, China)
}

\begin{abstract}
In the framework of the two-fluid model, a hypersonic flow of a nonuniform dusty gas with low inertial (non-depositing) particles around a blunt body is considered. The particle mass concentration is assumed to be small, so that the effect of particles on the carrier phase is significant only inside the boundary layer where the particles accumulate. Stepshaped and harmonic nonuniformities of the particle concentration ahead of the bow shock wave are considered and the corresponding nonstationary distributions of the particle concentration in the shock layer are studied. On the basis of numerical study of nonstationary two-phase boundary layer equations derived by the matched asymptotic expansion method, the effects of free-stream particle concentration nonuniformities on the thermal flux and the friction coefficient in the neighborhood of stagnation point are investigated, in particular, the most "dangerous" nonuniformity periods are found.
\end{abstract}

KEY WORDS: dusty gas, hypersonic flow, blunt body, non-stationary effects

\section{INTRODUCTION}

High-speed aircraft traveling in dusty atmosphere may meet aerosol or dust clouds with spatially nonuniform distribution of particles. In experimental studies of dusty-gas flow past blunt bodies in shock tubes or wind tunnels, it is very difficult to ensure the particle concentration uniformity during the testing time. These problems require a theoretical evaluation of nonstationary effects in the flow, induced by particle concentration nonuniformities ahead of the bow shock wave. It should be noted that investigations of dusty-gas flows past bodies are typical not only in connection with applications in high speed aerodynamics but also in certain technologies (spray-coating using two-phase jets, powder metallurgy, etc.). The presence of even small amount of particles in the free stream may drastically change the flow structure in the near wall region of a blunt body immersed in a high speed gas flow. For fairly large particles, the effect of surface erosion may be predominant (see, for example, Humphrey $\left.{ }^{[1]}\right)$. As was revealed in a series of experimental investigations in dust tunnels, ballistic ranges, and shock tubes with fairly inertial particles ${ }^{[2 \sim 6]}$; in hypersonic flows the

Received 31 December 1998

* The project supported by the Russian Foundation for Basic Research (project No. 96-01-00313) and the National Natural Science Foundation of China (joint RFBR-NSFC grant No.96-01-00017c) 
particles colliding with body surface may result in a sharp increase in the heat flux in the stagnation region of the body. Three basic physical mechanisms of heating augmentation are important in these conditions: turbulization of the boundary layer by particle wakes, additional turbulization due to the roughness of the body surface caused by impinging particles, and the main mechanism, the conversion of kinetic energy of the particles into thermal energy of the body. Low-inertial particles (less than several microns in size) usually do not deposit on the body surface. However, a theoretical analysis ${ }^{[7,8]}$ and experiments ${ }^{[9]}$ demonstrated that low-inertial particles may accumulate in the boundary layer on the up-wind surface of a blunt body, which also results in a noticeable change in the boundary layer structure and intensifies the heat transfer near the stagnation point even at very small particle concentrations in the free-stream. In this paper, we restrict our consideration to the regime of absence of particle inertial deposition on the body surface (low-inertial particles) and focus on nonstationary effects induced by particle concentration nonuniformities ahead of the bow shock wave.

\section{FORMULATION OF THE PROBLEM}

As the basis of the mathematical modeling, we use the two-fluid model of dusty gas with negligibly small volume fraction of particles ${ }^{[10]}$. Consider a plane or axisymmetric blunt body traveling at a supersonic velocity, which enters a layer of a nonuniform dusty gas at instant $t=0$. In the reference frame fitted to the body, nonstationarity of the problem is attributable to nonstationary conditions on the bow shock wave. We introduce a curvilinear coordinate system fitted to the stagnation point with the $x$ and the $y$ axes directed along and normal to the surface generatrix. We assume that the carrier phase is a perfect viscous gas and the particles are monodispersed spheres with the radius $\sigma$ and the mass $m$. Below, the subscript $s$ denotes the dispersed-phase parameters, the subscripts $\infty$ and $c$ refer to the free-stream and stagnation parameters, the asterisk signifies the dimensional variables where it is required to distinguish them from the corresponding nondimensional variables.

The expressions for the interphase momentum and energy exchange can be written as follows

$$
\begin{array}{ll}
f_{s}=6 \pi \sigma \mu^{*}\left(V^{*}-V_{s}^{*}\right) G & q_{s}=4 \pi \sigma \cdot \lambda^{*}\left(T^{*}-T_{s}^{*}\right) D \\
G=\left(1+\frac{1}{6} \operatorname{Re}_{s}^{2 / 3}\right)\left[1+\Phi_{1}\left(M_{s}, R e_{s}\right)\right] & D=\left(1+0.3 \operatorname{Pr}^{1 / 3} \operatorname{Re}_{s}^{1 / 2}\right) \Phi_{2}\left(M_{s}, R e_{s}\right)
\end{array}
$$

Here, $V^{*}$ is the velocity, $T^{*}$ is the temperature, $\mu^{*}$ and $\lambda^{*}$ are the gas viscosity and thermal conductivity, $\operatorname{Pr}$ is the Prandtl number of the gas, $R e_{s}$ and $M_{s}$ are the particle Reynolds and Mach numbers, the correction functions $\Phi_{1}, \Phi_{2}$ are taken in the form of Carlson and Hoglund ${ }^{[11]}$. For the temperature dependence of $\mu^{*}$ and $\lambda^{*}$ we assume the power law with the exponent $\omega$.

The nondimensional variables are introduced as follows

$$
\begin{aligned}
& x=\frac{x^{*}}{L} \quad y=\frac{y^{*}}{L} \quad \rho=\frac{\rho^{*}}{\rho_{\infty}^{*}} \quad n_{s}=\frac{n_{s}^{*}}{n_{s \infty}^{*}(0)} \quad \mu=\frac{\mu^{*}}{\mu_{c}^{*}} \\
& p=\frac{p^{*}}{\rho_{\infty}^{*} U_{\infty}^{2}} \quad T=\frac{2 T^{*} c_{p}}{U_{\infty}^{2}} \quad T_{s}=\frac{2 T_{s}^{*} c_{p}}{U_{\infty}^{2}} \quad t=\frac{t^{*} U_{\infty}}{L}
\end{aligned}
$$

Here, $n_{s}$ is the particle number concentration scaled to its value on the shock front on the symmetry axis at $t=0, L$ the radius of curvature of the body at the stagnation point, $U_{\infty}$ 
the free stream velocity, and $c_{p}$ the specific heat of the gas at constant pressure. Under the assumption of absence of 'crossing particle trajectories, the nondimensional equations of dusty gas flow take the form

$$
\begin{aligned}
& \frac{\partial \rho}{\partial t}+\operatorname{div}(\rho V)=0 \quad \quad \frac{\partial n_{s}}{\partial t}+\operatorname{div}\left(n_{s} V_{s}\right)=0 \\
& \rho \frac{\partial \boldsymbol{V}}{\partial t}+\rho(\boldsymbol{V} \nabla) \boldsymbol{V}+\nabla p+\alpha \beta \mu n_{s}\left(\boldsymbol{V}-\boldsymbol{V}_{s}\right) G=\frac{2 \varepsilon}{3 \kappa}[-\nabla(\mu \operatorname{div} \boldsymbol{V})+3 \operatorname{div} \mu \boldsymbol{S}] \\
& \frac{\partial V_{s}}{\partial t}+\left(V_{s} \nabla\right) V_{s}=\beta \mu G\left(V-V_{s}\right) \\
& \frac{\partial T_{s}}{\partial t}+\left(V_{s} \nabla\right) T_{s}=\frac{2 c_{p}}{3 c_{s} P r} \beta \mu D\left(T-T_{s}\right) \\
& \frac{\partial T}{\partial t}+\rho(V \nabla) T=2(V \nabla) p+\frac{2 \varepsilon}{\kappa}\left[2 \mu S^{2}-\frac{2}{3} \mu(\operatorname{div} V)^{2}\right]+\frac{\varepsilon}{\kappa P r} \operatorname{div}(\mu \nabla T)+ \\
& 2 \alpha \beta \mu n_{s} G\left|V-V_{s}\right|^{2}+\frac{2}{3} \frac{\alpha \beta \mu}{P r} n_{s} D\left(T_{s}-T\right) \\
& p=\frac{\gamma-1}{2 \gamma} \rho T \quad \mu=T^{\omega} \quad \kappa=\frac{\gamma-1}{\gamma+1} \quad \operatorname{Pr}=\frac{c_{p} \mu^{*}}{\lambda^{*}} \\
& \left.\alpha=\frac{m n_{s \infty}^{*}}{\rho_{\infty}^{*}} \quad \varepsilon=\frac{1}{R e}=\frac{\mu_{c}^{*}}{U_{\infty} \rho_{c}^{*} L} \quad \beta=\frac{6 \pi \sigma \mu_{c}^{*} L}{m U_{\infty}} \quad \rho_{c}^{*}=\frac{\rho_{\infty}^{*}}{\kappa}\right)
\end{aligned}
$$

Here, $\gamma$ is the specific heat ratio, $1 / \kappa$ the gas compression at the hypersonic velocity, $\boldsymbol{S}$ the strain rate tensor, and $c_{s}$ the specific heat of the particle material.

For the carrier phase, on the body surface the no-slip condition and a fixed wall temperature $T_{w}$ are specified. Ahead of the bow shock wave the flow velocity and temperature are assumed to be constant while the particle concentration is nonuniform in the flow direction. This is the reason of the occurrence of nonstationarities in the shock and the boundary layers. Hereafter we consider step-shaped and harmonic nonuniformities of the particle concentration.

We assume that the flow Reynolds and Mach numbers are high $\left(\varepsilon \ll 1, M_{\infty}^{2} \gg 1\right)$ and the particle mass concentration is small $(\alpha \ll 1)$. These assumptions make it possible to simplify significantly the problem formulation: when $\alpha \rightarrow 0$, outside the boundary layer the effect of particles on the carrier phase vanishes and hence all the flow parameters are timeindependent except for the particle concentration. For gas parameters, in the inviscid region of the shock layer we can use the well-known hypersonic solutions ${ }^{[12,13]}$ and the dispersedphase equations in Lagrange variables can be reduced to ordinary differential equations on a fixed particle trajectory ${ }^{[14]}$. This makes it possible to investigate the nonstationary behavior of the particle concentration in the shock layer and then to use it as the outer solution for the nonstationary boundary layer flow.

The other restriction is that in what follows we consider only the regime of absence of particle inertial deposition, i.e. the case of sufficiently small particles. This regime takes place when the particle inertia parameter is greater than a certain threshold value obtained by Osiptsov and Shapiro ${ }^{[8]}: \beta / u_{1} \geq \beta^{0}\left(R e_{s 0}\right)$ where $R e_{s 0}=2 \sigma \rho_{c}^{*} U_{\infty} / \mu_{c}^{*}$ and $u_{1}=$ $\partial u_{e} / \partial x(0,0)$ is the nondimensional velocity gradient of the inviscid flow at the stagnation point. 


\section{INVISCID FLOW REGION}

As an example of a blunt body, we consider a sphere of radius $L$, for which the hypersonic solution for gas parameters in the shock layer ${ }^{[12]}$ can be written in the nondimensional form as follows

$$
\begin{aligned}
& u(x, y)=\left(\frac{y}{\kappa}+\sqrt{\frac{8 \kappa}{3}}-4 y\right) \sin (x) \\
& v(x, y)=-\cos (x)\left[\frac{y^{2}}{\kappa}+2 y \sqrt{\frac{8 \kappa}{3}}-4 y^{2}\left(1+\frac{y}{3 \kappa}\right)\right] \\
& T=1+\frac{2}{(\gamma-1) M_{\infty}^{2}}-u^{2}-v^{2} \quad \rho=\frac{1}{\kappa} \\
& 0 \leq y \leq y_{s h} \quad y_{s h}=\kappa\left(1-\sqrt{\frac{8 \kappa}{3}}+4 \kappa\right)
\end{aligned}
$$

This is an asymptotic solution $\left(M_{\infty}^{2} \gg 1, \kappa \ll 1\right)$ correct to the terms of the order of $\kappa^{2 / 3}$. In the expression for the temperature we retain the higher-order terms which are important near the symmetry axis.

It is clear that, since the boundary conditions for the particle velocity and temperature on the shock front are time-independent, these parameters are steady-state and equal to the steady-state flow parameters obtained for the constant particle concentration in the free stream. In the inviscid shock layer the only nonstationary parameter is the particle concentration for which, on a fixed particle trajectory, the following relationship is valid

$$
\frac{\mathrm{d}}{\mathrm{d} \tau} \ln n_{s}=-\operatorname{div} V_{s}
$$

From this it follows

$$
n_{s}\left(\tau, x_{0}\right)=n_{s}\left(0, x_{0}\right) \exp \left(-\int_{0}^{\tau} \operatorname{div} V_{s} \mathrm{~d} t\right)
$$

Here, $x_{0}$ is the abscissa of the origin of a particle trajectory on the bow shock wave (at $y=y_{s h}$ ), and $\tau$ is the nondimensional time of the particle motion along this trajectory. Since the particle velocity field is time-independent, it follows from this equation that a particle concentration nonuniformity is transported at the dispersed-phase velocity, with the nonuniformity amplitude depending on the deformation of an elementary Lagrange volume of the dispersed phase. Equation (3.1) can be rewritten in the following form

$$
n_{s}\left(t, x_{0}\right)=n_{s f}\left(t-\tau, x_{0}\right) n_{s u}\left(\tau, x_{0}\right)
$$

Here, $t$ is measured from the instant at which the leading point of the shock front enters the concentration nonuniformity, $n_{s f}$ is the concentration on the shock front, and $n_{s u}$ is the particle concentration distribution in the shock layer in the case of the uniform particle distribution ahead of the shock wave. Relation (3.2) in fact reduces the nonstationary problem to the steady state one: to calculate the particle concentration in the nonstationary problem at a fixed point of the shock layer one must calculate $n_{s u}$ from the steady-state problem, find the time of particle motion along the trajectory from the wave surface to the considered point $(\tau)$, and determine the particle concentration on this trajectory on the shock front at the instant $t-\tau\left(n_{s f}\left(t-\tau, x_{0}\right)\right)$ for a given particle distribution ahead of the shock front. 
The steady-state problem is solved in the Lagrangian variables $x_{0}, \tau$, in which the equations of motion and energy of the dispersed phase take the form

$$
\begin{aligned}
\frac{\mathrm{d} x_{s}}{\mathrm{~d} \tau}=\frac{u_{s}}{1+y_{s}} \quad \frac{\mathrm{d} y_{s}}{\mathrm{~d} \tau}=v_{s} & \frac{\mathrm{d} u_{s}}{\mathrm{~d} \tau}=\beta \mu G\left(u-u_{s}\right)-\frac{u_{s} v_{s}}{1+y_{s}} \\
\frac{\mathrm{d} v_{s}}{\mathrm{~d} \tau}=\beta \mu G\left(v-v_{s}\right)+\frac{u_{s}^{2}}{1+y_{s}} & \frac{\mathrm{d} T_{s}}{\mathrm{~d} \tau}=\frac{2 \beta c_{p}}{3 c_{s} P r} \mu D\left(T-T_{s}\right)
\end{aligned}
$$

In the Lagrange form, the continuity equation for the steady-state flow with the uniform particle distribution ahead of the bow shock can be written as follows

$$
\frac{1}{n_{s}\left(\tau, x_{0}\right)}=\frac{\left(1+y_{s}\right) \sin \left(x_{s}\right)\left[u_{s} w_{3} / \kappa-v_{s} w_{1}\left(1+y_{s}\right)\right]}{\left(1+y_{s h}\right)^{2} \sin \left(x_{0}\right) \cos \left(x_{0}\right)}
$$

To use this equation for calculating the particle concentration along a fixed particle trajectory, we introduce the following additional variables

$$
w_{1}=\frac{\partial x_{s}\left(\tau, x_{0}\right)}{\partial x_{0}} \quad w_{2}=\frac{\partial u_{s}\left(\tau, x_{0}\right)}{\partial x_{0}} \quad w_{3}=\frac{\partial y_{s}\left(\tau, x_{0}\right)}{\partial x_{0}} \quad w_{4}=\frac{\partial v_{s}\left(\tau, x_{0}\right)}{\partial x_{0}}
$$

The equations for these variables are derived by differentiating the equations of particle motion with respect to $x_{0}$

$$
\begin{aligned}
& \frac{\mathrm{d} w_{1}}{\mathrm{~d} \tau}=\frac{w_{2}}{1+y_{s}}-\frac{u_{s} w_{3}}{\left(1+y_{s}\right)^{2}} \\
& \frac{\mathrm{d} w_{2}}{\mathrm{~d} \tau}=\beta \mu G\left(w_{1} \frac{\partial u}{\partial x}+w_{3} \frac{\partial u}{\partial y}-w_{2}\right)+\beta\left(u-u_{s}\right) \frac{\partial}{\partial x_{0}}(G \mu)-\frac{u_{s} w_{4}+v_{s} w_{2}}{1+y_{s}}+\frac{u_{s} v_{s} w_{3}}{\left(1+y_{s}\right)^{2}} \\
& \frac{\mathrm{d} w_{3}}{\mathrm{~d} \tau}=w_{4} \\
& \frac{\mathrm{d} w_{4}}{\mathrm{~d} \tau}=\beta \mu G\left(w_{1} \frac{\partial v}{\partial x}+w_{3} \frac{\partial v}{\partial y}-w_{4}\right)+\beta\left(v-v_{s}\right) \frac{\partial}{\partial x_{0}}(G \mu)+\frac{2 u_{s} w_{2}}{1+y_{s}}-\frac{u_{s}^{2} w_{3}}{\left(1+y_{s}\right)^{2}}
\end{aligned}
$$

These equations together with the particle motion equations constitute a system of ordinary differential equations along a fixed particle trajectory, which makes it possible to calculate all the dispersed-phase parameters, including the concentration. The expression for $\partial(\mu G) / \partial x_{0}$ is cumbersoine and not reproduced here.

The conditions on the shock wave follow from the continuity of particle parameters on the shock front

$$
\begin{array}{lllll}
\tau=0: & x_{s}=x_{0} & y_{s}=y_{s h} & u_{s}=\sin \left(x_{0}\right) & v_{s}=-\cos \left(x_{0}\right) \\
T_{s}=T_{\infty} & w_{1}=1 & w_{2}=\cos \left(x_{0}\right) & w_{3}=0 & w_{4}=\sin \left(x_{0}\right)
\end{array}
$$

Detailed numerical calculations of the steady-state problem for a sphere travelling in the uniform dusty-gas flow were performed in Osiptsov and Shapiro ${ }^{[15]}$ for both the regimes of presence and absence of particle inertial deposition on the body surface.

The particle distribution in the shock layer depends on the two nondimensional parameters $\beta_{0}=\beta / u_{1}$ and $R b=R e_{s}^{2 / 3} / 6$ only. It follows from the numerical calculations that with decrease in particle inertia (increase in the parameter $\beta_{0}$ ), once a certain threshold value $\beta^{0}(R b)$ is passed, the particles no longer deposit on the body surface. 


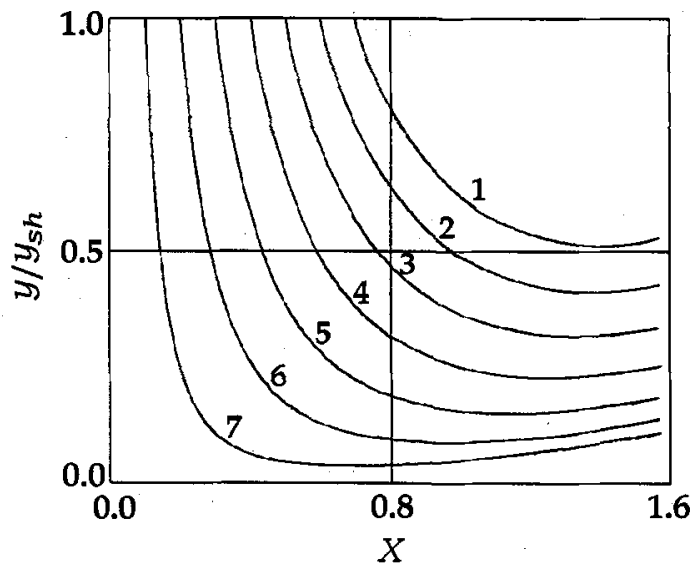

Fig.1 Particle trajectories for $\beta_{0}=8.1$, $R b=2.5:$ curves $1 \sim 7$ correspond to $x_{0}=0.7 \sim 0.1$

In Fig.2, the dashed curve shows a typical steady-state profile of the particle concentration in the shock layer for the uniform free-stream distribution of the particles. For comparison, in the same figure we plotted the particle concentration profile corresponding to $t=4$ for the step-shaped concentration nonuniformity in the free stream, with the step amplitude equal to 0.9 and the step length equal to 0.5. Clearly, the amplitude of the concentration nonuniformity sharply increases with the decrease of the distance from the wall. This follows from the fact that on the symmetry axis the time of motion of a marked particle towards the wall is infinite and the distance between any two marked particles tends to zero with time. Hence, no matter how small is the initial difference in the concentration at the locations of the marked particles, as the time increases, this
Therewith, the particle concentration grows unboundedly near the limiting (the nearest to the surface) particle trajectory which, due to the difference in inertial properties of the phases, does not coincide with the front surface of the body. For $R b \geq 2.5$, for axisymmetric and plane flows the threshold value of $\beta^{0}$ is equal to 8 and 4 , respectively. In what follows we will consider the near-critical flow regime with absence of the particle deposition. For this case, the typical pattern of the particle trajectories is represented in Fig.1. It follows from the calculations that in the no-deposition regime a very thin particle-free region arises between the limiting particle trajectory and the body surface.

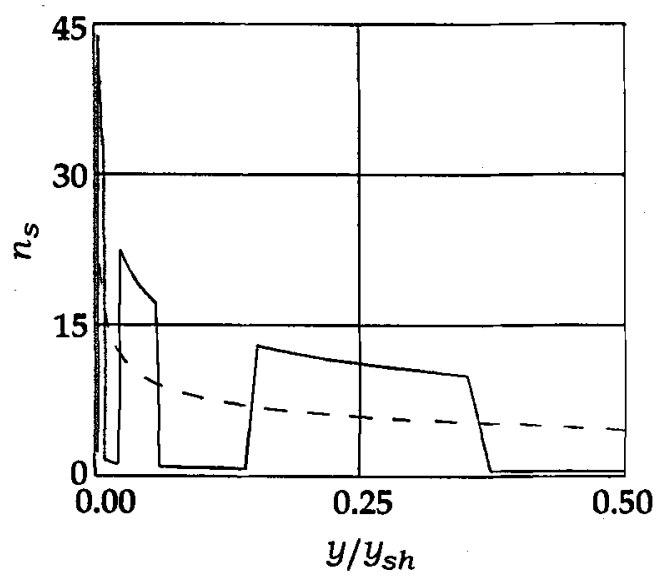

Fig.2 Propagation of the step-shaped nonuniformity in the shock layer. Dashed curve: uniform distribution in the free stream; Solid curve: step-shaped nonuniformity with the initial step amplitude equal to 0.9 and length equal to $0.5(t=4, \beta=$ $8.1, R b=2.5)$

concentration difference tends to infinity. It is also clear that the concentration distribution for the nonuniform flow lies between the concentration profiles for both the uniform flows with free-stream concentrations equal to the maximum and the minimum values of the concentration nonuniformity in the free stream.

Figure 3 presents the successive locations of the leading front of the nonuniformity in the shock layer vs. time for $\beta_{0}=8.1$ and $R b=2.5$, curves $1 \sim 6$ correspond to $t=$ $0.01,0.1,0.5,1.0,1.5,2.0$. As is clear, as the time increases, a fixed Lagrangian volume of the dispersed phase is compressed in the $y$ direction and extends in the $x$ direction, therewith 
the nonuniformity front does not reach the frontal surface of the body at any finite time interval. It is important to find the dimensions of the free-stream Lagrangian region which can simultaneously enter the shock layer and hence determine the current distribution of the particle concentration in the shock layer.

In Fig.4, in the cylinder coordinates scaled to $L$ and fitted to the leading point of the shock wave $\left(z_{1}\right.$ is directed opposite the free stream and $r_{1}$ is the distance from the symmetry axis) we present the calculated boundaries of such free-stream region which at instant $t=4$ occupies almost the entire shock layer $(0 \leq x \leq 1)$.

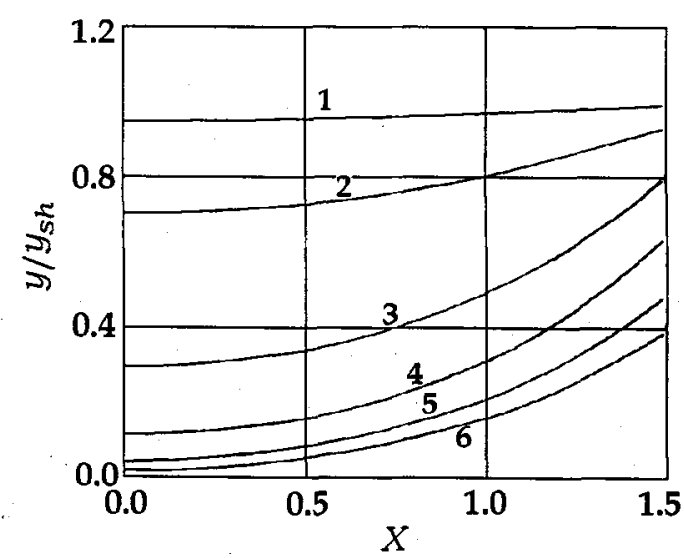

Fig.3 Propagation of the nonuniformity front in the shock layer. Curves $1 \sim 6$ correspond to $t=0.01,0.1,0.5,1.0,1.5,2.0$; $\beta_{0}=8.1, R b=2.5$

In Fig.5, we plotted the particle concentration distribution $(t=4)$ in the nearwall region on the symmetry axis for the case of harmonic free-stream nonuniformity with the period equal to $\pi L / 6$ and the amplitude equal to 0.3 . Clearly, with decrease in distance from the wall the concentration increases unboundedly, the amplitude of the nonuniformity also increases and the period sharply decreases.

Such behavior of the particle concentration poses a problem for the effect of particles on the boundary layer structure and the possibility of appearance of peak heat fluxes to the body surface. To derive the boundary layer equations, we must know the asymptotic behavior of the particle parameters obtained in inviscid problem as $y \rightarrow 0$.

An asymptotic analysis of the behavior

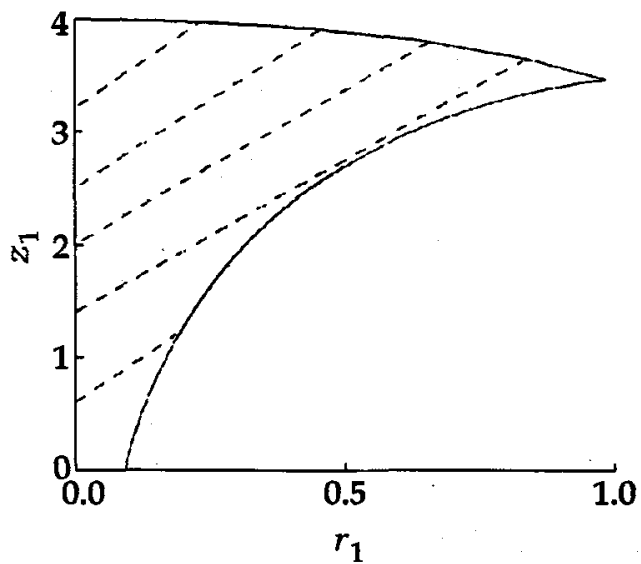

Fig.4 Initial Lagrangian region in the cylinder coordinates which occupies the shock layer at $t=4, \beta_{0}=8.1, R b=2.5$

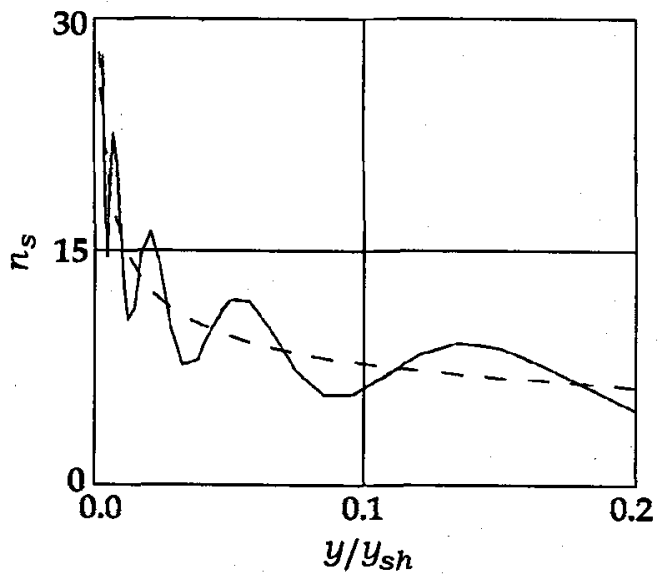

Fig.5 Particle concentration distribution near the wall on the symmetry axis at $t=4$ for harmonic free-stream concentration profile (amplitude $=0.3$, period $=\pi L / 6$ ). Dashed curve - concentration distribution in the uniform flow $\left(\beta_{0}=8.1, R b=\right.$ 2.5) 
of the dispersed-phase parameters in the neighborhood of a potential-flow stagnation point ${ }^{[16]}$ indicated that in the exact steady-state solution the singularity of $n_{s}$ has the type $n_{s} \sim 1 / y^{r}$ where $r$ varies over the range $0 \leq r \leq 2-\sqrt{2}$ (plane flow) or $0 \leq r \leq 3-\sqrt{6}$ (axisymmetric flow). The concentration singularity is integrable and hence the mean distance between the particles remains finite and the model of noncolliding particles is still valid ${ }^{[16]}$. The analysis and the calculations carried out by Osiptsov and Shapiro ${ }^{[15]}$ demonstrated that if the particle concentration is represented as $n_{s}=d(x) / Y_{1}^{r}+\ldots$ where $Y_{1}$ is the nondimensional distance from the limiting particle trajectory, for $x \leq 1$ the function $d(x)$ is almost constant, equal to $d(0)$ which is a function of $\beta_{0}$ and $R b$. In Osiptsov and Shapiro ${ }^{[8]}$, the value of $d(0)$ was calculated over a wide range of $\beta_{0}$ and $R b$.

We will now determine the near-wall asymptotics of the particle concentration for the nonstationary problem on the symmetry axis. Using relation (3.2), for $y \ll 1$ we can write

$$
n_{s}(t, y)=n_{s f}(t-\tau) \frac{d(0)}{y^{r}}
$$

Now we will evaluate the time $\tau$ which it takes for the nonuniformity front to reach the near wall region. In the neighborhood of the stagnation point, for small $y$ we can write

$$
u=u_{1} x \quad v=-2^{j} u_{1} y \quad v_{s}=v_{s 1} y
$$

where

$$
v_{s 1}=-\frac{\beta}{2}+\sqrt{\frac{\beta^{2}}{4}-2^{j} \beta u_{1}}
$$

Then, for small $y$ we obtain

$$
\tau=\text { const }+\frac{1}{v_{s 1}} \ln y
$$

and hence the asymptotics of the particle concentration takes the form

$$
n_{s}(t, y)=\frac{d(0)}{y^{r}} n_{s f}(\varphi) \quad \varphi=t-\frac{1}{v_{s 1}} \ln y-\text { const }
$$

This asymptotics will be used as the matching condition with the particle concentration in the boundary layer. From Eq.(3.3) it is clear that the concentration nonuniformity front reaches the outer edge of the boundary layer at large values of $t$.

The particle accumulation in the near wall region in the no-deposition regime allows us to assume that inside the boundary layer the effect of particles on the carrier phase may be considerable even at very low mass concentrations $\alpha$ in the free stream. Below we investigate the boundary layer structure in the stagnation point neighborhood in the regime of no inertial deposition of particles.

\section{NONSTATIONARY BOUNDARY LAYER NEAR THE STAGNATION POINT}

To derive the boundary layer equations we use the technique of the matched asymptotic expansions ${ }^{[17]}$. A review on mathematical modeling of dusty-gas boundary layers can be found in Osiptsov ${ }^{[18]}$, in particular, the steady-state boundary layer at the stagnation point 
of a body in a uniform hypersonic dusty-gas flow was studied in Osiptsov and Shapiro ${ }^{[8]}$. Below, we consider the nonstationary two-phase boundary layer on a front surface of a body with finite curvature and restrict our consideration to the regime of absence of inertial deposition of particles $\beta_{0} \geq \beta^{0}$. The other limitation is that we consider only a neighborhood of the stagnation point, a region $X=x / \varepsilon_{1}^{1 / 4} \sim O(1)$ (here, $\varepsilon_{1}=\varepsilon / \kappa$ ), since for $x \sim O(1)$ due to noncoincidence of the body surface and the limiting trajectory the particle trajectories do not enter the boundary layer from the outer flow.

We assume that in the no-deposition regime the leading terms of the outer (inviscid) solution in this region near the body surface $(\varepsilon \rightarrow 0, \alpha \rightarrow 0, X$ is fixed and $y \rightarrow 0)$ is known and can be represented in the form (denoted by subscript $e$ )

$$
\begin{array}{lcc}
v_{e}=v_{s e}=0 & \\
n_{s e}(X, y, t) \sim d(X) n_{s f}(X, \varphi) / y^{r} & d(X) \sim O(1) \\
\varepsilon_{1}^{1 / 4} u_{e}(X) & \varepsilon_{1}^{1 / 4} u_{s e}(X) & p_{e}=p_{c}+\sqrt{\varepsilon_{1}} p_{e 1}(X) \\
\rho_{e}(X) & T_{e}(X) & T_{s e}(X) \sim O(1)
\end{array}
$$

To derive the boundary layer equations we introduce a stretched boundary-layer coordinate $\eta=y / \sqrt{\varepsilon_{1}}$ and a new "inner" time

$$
t_{i}=t-\frac{1}{2 v_{s 1}} \ln \varepsilon_{1}
$$

The expression for the inner time follows from the fact that at a fixed $X$ matching the outer and inner expansions of the particle concentration can be most easily carried out in the variables $(y, \varphi)$ and $(\eta, \varphi)$ as $y \rightarrow 0$ and $\eta \rightarrow \infty$, respectively, when the value of $\varphi$ is fixed: The condition of coincidence of the expression for $\varphi$ in the outer variables $(t, y)$ and the inner variables $\left(t_{i}, \eta\right)$ gives the required expression for $t_{i}$. We note that the matching at a fixed $\varphi$ means that the outer coordinate tends to zero $(y \rightarrow 0)$ and the outer time tends to infinity $t \rightarrow \infty$ while the inner coordinate and the inner time tend to plus and minus infinity, respectively $(\eta \rightarrow \infty, t \rightarrow-\infty)$. We will seek the inner solution in the boundary layer in the form of series in $\varepsilon_{1}$. For the normal velocities of the phases and the particle concentration the leading terms (denoted by subscript 0 ) are

$$
\sqrt{\varepsilon_{1}} v_{0}\left(X, \eta, t_{i}\right) \quad \sqrt{\varepsilon_{1}} v_{s 0}\left(X, \eta, t_{i}\right) \quad d(X) n_{s 0}\left(X, \eta, t_{i}\right) / \varepsilon_{1}^{r / 2}
$$

For the longitudinal velocity components, the leading terms are

$$
\varepsilon_{1}^{1 / 4} u_{0}\left(X, \eta, t_{i}\right) \quad \varepsilon_{1}^{1 / 4} u_{s 0}\left(X, \eta, t_{i}\right)
$$

For other variables the leading terms are of the order of unity. As is usually in the matched asymptotic expansion method, the orders of the quantities in the expansions are chosen from the conditions of matching with the outer solution. Substituting the inner expansions in (3.1) and retaining the leading terms, we obtain the boundary layer equations 
for the no-deposition regime on the frontal surface of a body with finite curvature

$$
\begin{aligned}
& \frac{\partial \rho_{0}}{\partial t_{i}}+\frac{\partial \rho_{0} u_{0} X^{j}}{\partial X}+\frac{\partial \rho_{0} v_{0} X^{j}}{\partial \eta}=0 \\
& \frac{\partial n_{s 0}}{\partial t_{i}}+\frac{\partial n_{s 0} u_{s 0} X^{j}}{\partial X}+\frac{\partial n_{s 0} v_{s 0} X^{j}}{\partial \eta}=0 \\
& \rho_{0}\left(\frac{\partial u_{0}}{\partial t_{i}}+u_{0} \frac{\partial u_{0}}{\partial X}+v_{0} \frac{\partial u_{0}}{\partial \eta}\right)+\frac{\alpha \beta}{\varepsilon_{1}^{r / 2}} \mathrm{~d} \mu_{0} n_{s 0}\left(u_{0}-u_{s 0}\right)= \\
& \frac{\partial}{\partial \eta}\left(\mu_{0} \frac{\partial u_{0}}{\partial \eta}\right)-\frac{\mathrm{d} p_{e 1}}{\mathrm{~d} X} \\
& \frac{\partial p_{0}}{\partial \eta}=0 \quad p_{c}=\frac{\gamma-1}{2 \gamma} \rho_{0} T_{0} \\
& \frac{\partial u_{s 0}}{\partial t_{i}}+u_{s 0} \frac{\partial u_{s 0}}{\partial X}+v_{s 0} \frac{\partial u_{s 0}}{\partial \eta}=\beta \mu_{0}\left(u_{0}-u_{s 0}\right) \\
& \frac{\partial v_{s 0}}{\partial t_{i}}+u_{s 0} \frac{\partial v_{s 0}}{\partial X}+v_{s 0} \frac{\partial v_{s 0}}{\partial \eta}=\beta \mu_{0}\left(v_{0}-v_{s 0}\right)+u_{s 0}^{2} \\
& \frac{\partial T_{s 0}}{\partial t_{i}}+u_{s 0} \frac{\partial T_{s 0}}{\partial X}+v_{s 0} \frac{\partial T_{s 0}}{\partial \eta}=\frac{2 c_{p} \beta}{3 c_{s} P r} \mu_{0}\left(T_{0}-T_{s 0}\right) \\
& \frac{\partial T_{0}}{\partial t_{i}}+\rho_{0}\left(u_{0} \frac{\partial T_{0}}{\partial X}+v_{0} \frac{\partial T_{0}}{\partial \eta}\right)=\frac{1}{P r} \frac{\partial}{\partial \eta}\left(\mu_{0} \frac{\partial T_{0}}{\partial \eta}\right)+ \\
& \frac{2 \alpha \beta}{3 \varepsilon_{1}^{r / 2} P r} \mathrm{~d} \mu_{0} n_{s 0}\left(T_{s 0}-T_{0}\right) \\
& \mu_{0}=T_{0}^{\omega} \quad
\end{aligned}
$$

The boundary and matching conditions take the form

$$
\begin{array}{lll}
\eta=0: & u_{0}=v_{0}=0 & T_{0}=T_{w} \\
\eta \rightarrow \infty: & u_{0} \rightarrow u_{e} & u_{s 0} \rightarrow u_{s e} \\
T_{0} \rightarrow T_{e} & T_{s 0} \rightarrow T_{s e} & \left.\frac{\partial v_{s 0}}{\partial \eta} \rightarrow \frac{\partial v_{s e}}{\partial y}\right|_{y=0} \\
\eta \rightarrow \infty, t_{i} \rightarrow-\infty(\varphi=\text { const }): & \eta^{r} n_{s 0} \rightarrow n_{s f}(X, \varphi)
\end{array}
$$

From Eqs.(4.1), it is clear that the source terms describing the effect of particles on the carrier phase in the boundary layer are of the order of $\alpha / \varepsilon_{1}^{r / 2}$ and hence this effect is significant even at small $\alpha$. To obtain the quantitative estimate of this effect for nonuniform free-stream profiles of the particle concentration, we consider a local solution of Eqs.(4.1) in a small neighborhood of the stagnation point where the outer solution can be represented in the form (subscript 1 refers to the leading coefficients)

$$
\begin{aligned}
& u_{e}=u_{1} X \quad v_{e}=-2^{j} u_{1} y \\
& u_{s e}=u_{s 1} X \quad v_{s e}=v_{s 1} y \\
& T_{e}=1+\cdots \quad T_{s e}=1+\cdots \quad n_{s e}=d(0) n_{s f}(\varphi) / y^{r / 2}
\end{aligned}
$$


Vol.15, No.3

We seek the local solution of Eqs.(4.1) near the stagnation point in the form

$$
\begin{array}{ll}
u_{0}=u_{1} X f\left(t_{i}, z\right)+\cdots & v_{0}=-u_{1} \eta g\left(t_{i}, z\right)+\cdots \\
u_{s 0}=u_{s 1} X f_{s}\left(t_{i}, z\right)+\cdots & v_{s 0}=v_{s 1} \eta g_{s}\left(t_{i}, z\right)+\cdots \\
T_{0}=\theta\left(t_{i}, z\right)+\cdots & T_{s 0}=\theta_{s}\left(t_{i}, z\right)+\cdots \\
\rho_{0}=\psi\left(t_{i}, z\right) / g\left(t_{i}, z\right) & n_{s 0}=\psi_{s}\left(t_{i}, z\right) /\left(\eta^{r} g_{s}\right)+\cdots
\end{array}
$$

Here, $z=\eta \sqrt{u_{1}}$. Substituting these expansions in Eqs.(4.1), we obtain the following problem

$$
\begin{aligned}
& \frac{\psi}{u_{1} g} \frac{\partial f}{\partial t_{i}}-\theta^{\omega} \frac{\mathrm{d}^{2} f}{\mathrm{~d} z^{2}}-\omega \theta^{\omega-1} \frac{\mathrm{d} f}{\mathrm{~d} z} \frac{\mathrm{d} \theta}{\mathrm{d} z}+\frac{\psi f^{2}}{g}-z \psi \frac{\mathrm{d} f}{\mathrm{~d} z}-\frac{1}{\kappa}+ \\
& A \beta_{0} \theta^{\omega}\left(f-a f_{s}\right) \frac{\psi_{s}}{z^{r} g_{s}}=0 \\
& \frac{1}{u_{1}} \frac{\partial}{\partial t_{i}}\left(\frac{\psi}{g}\right)-z \frac{\mathrm{d} \psi}{\mathrm{d} z}+\frac{2^{j} f \psi}{g}-\psi=0 \\
& \frac{1}{u_{1}} \frac{\partial}{\partial t_{i}}\left(\frac{\psi_{s}}{g_{s}}\right)-c z \frac{\mathrm{d} \psi_{s}}{\mathrm{~d} z}+\frac{2^{j} a f_{s} \psi_{s}}{g_{s}}+c(r-1) \psi_{s}=0 \\
& \frac{1}{u_{1}} \frac{\psi}{g} \frac{\partial \theta}{\partial t_{i}}-\frac{\theta^{\omega}}{\operatorname{Pr}} \frac{\mathrm{d}^{2} \theta}{\mathrm{d} z^{2}}-\omega \theta^{\omega-1}\left(\frac{\mathrm{d} \theta}{\mathrm{d} z}\right)^{2}-\frac{2 A \beta_{0}}{3 P r} \psi_{s} \theta^{\omega} \frac{\theta_{s}-\theta}{g_{s} z^{r}}-z \psi \frac{\mathrm{d} \theta}{\mathrm{d} z}=0 \\
& \frac{a}{u_{1}} \frac{\partial f_{s}}{\partial t_{i}}-a c z g_{s} \frac{\mathrm{d} f_{s}}{\mathrm{~d} z}+\left(a f_{s}\right)^{2}-\beta_{0} \theta^{\omega}\left(f-a f_{s}\right)=0 \\
& \frac{c}{u_{1}} \frac{\partial g_{s}}{\partial t_{i}}-c^{2} z g_{s} \frac{\mathrm{d} g_{s}}{\mathrm{~d} z}+\beta_{0} \theta^{\omega}\left(c g_{s}-g\right)-\left(c g_{s}\right)^{2}=0 \\
& \frac{1}{u_{1}} \frac{\partial \theta_{s}}{\partial t_{i}}-c z g_{s} \frac{\mathrm{d} \theta_{s}}{\mathrm{~d} z}-\frac{2}{3} \frac{c_{p}}{c_{s}} \frac{\beta_{0} \theta^{\omega}}{\operatorname{Pr}}\left(\theta-\theta_{s}\right)=0 \\
& \frac{1}{\kappa}=\frac{\theta \psi}{g} \quad A=\frac{\alpha d(0) u_{1}^{r / 2}}{\varepsilon_{1}^{r / 2}} \quad a=\frac{u_{s 1}}{u_{1}} \quad c=-\frac{v_{s 1}}{u_{1}} \\
& z=0: \quad f=\psi=0 \quad \theta=T_{w} \\
& z=\infty: \quad f=f_{s}=g_{s}=\theta=\theta_{s}=1 \\
& z \rightarrow \infty, t_{i} \rightarrow-\infty(\varphi=\text { const }): \psi_{s} \rightarrow n_{s f}(\varphi)
\end{aligned}
$$

Since the parameters $r, a$ and $c$ are functions of $\beta_{0}{ }^{[7]}$

$$
r=1-2^{j} \frac{a}{c} \quad a=-\frac{\beta_{0}}{2}+\sqrt{\frac{\beta_{0}^{2}}{4}+\beta_{0}} \quad c=\frac{\beta_{0}}{2}-\sqrt{\frac{\beta_{0}^{2}}{4}-2^{j} \beta_{0}}
$$

for a fixed gas (fixed $P r, \gamma$, and $\omega$ ) the problem (4.2) depends on the function $n_{s f}(\varphi)$ and the following similarity criteria: $A, \beta_{0}, c_{s} / c_{p}$ and $T_{w}$.

We consider two types of the nonuniformities, the first one described by the formulas

$$
t<0: \quad n_{s f}=1 \quad t \geq 0: \quad n_{s f}(t)=2-\cos (2 \pi \Omega t)
$$

and the second, described by the same formulas at $t<1 / 4 \Omega$ and for $t \geq 1 / 4 \Omega: \quad n_{s f}=2$. Here, $\Omega$ characterizes the ratio of the body scale $L$ and the nonuniformity period. 
Equations (4.2) were calculated numerically by a finite-difference method. For convenience, the integration domain with respect to $z$ coordinate was transformed into the interval $[0,1]$ using the change of the variable: $z_{1}=(2 / \pi) \operatorname{arctg}(\pi z / 2)$. In the calculations, it was assumed: $\gamma=1.4, \operatorname{Pr}=0.7$ and $\omega=0.5$.

In the first stage, we calculated the steady-state distributions of the parameters corresponding to the uniform $\left(n_{s f}(\varphi)=1\right)$ particle concentration on the outer edge of the boundary layer. We used the same finite-difference method with iterations with respect to the terms describing the momentum and energy exchange as in Osiptsov and Shapiro $(1986)^{[8]}$. This solution gives the distributions of the parameters in the initial section of the calculation domain. Since the problem (4.2) is parabolic with respect to coordinate $t_{i}$, we used a cruise implicit method with respect to $t_{i}$ and the sweep method at each time step. In the calculations the boundary conditions at infinity were in fact brought down to a very large but finite value of $z$, and the negative value of $t_{i}$ at which the nonuniformity front reaches this point was taken as the origin of a drifted inner time and this section was taken as the initial section of the calculation domain $t_{i}=0$. The possibility of the drift of the inner time origin is connected with the presence of an arbitrary constant in the definition of $\varphi$.

In Fig.6, for different values of $\beta_{0}$ and $c_{p} / c_{s}=1$ we present the relative steady-state $\left(n_{s f}=1\right)$ thermal flux $\left(Q / Q_{0}\right)$ and friction coefficient $\left(C_{f} / C_{f 0}\right)$ vs. the parameter $A$ characterizing the magnitude of the particle effect on the carrier-phase parameters, here the subscript 0 refers to the corresponding values in the pure gas. As is clear, the maximal effect of particles on the boundary layer structure is attained for values $\beta_{0}$ near the limit of the no-deposition regime $\left(\beta_{0}=8.01\right)$. With increase in $\beta$ (decrease in the particle inertia), the effect of heating augmentation by particles decreases. Over the range of parameter $A$ considered, the effect of particles on the friction is fairly small. The reason for the particle effect on the heat flux to the stagnation point is that inside the boundary layer the particles, heated in the inviscid region of the shock layer, work as heat sources in the carrier phase.

The intensity of these sources is proportional to the local mass concentration of the

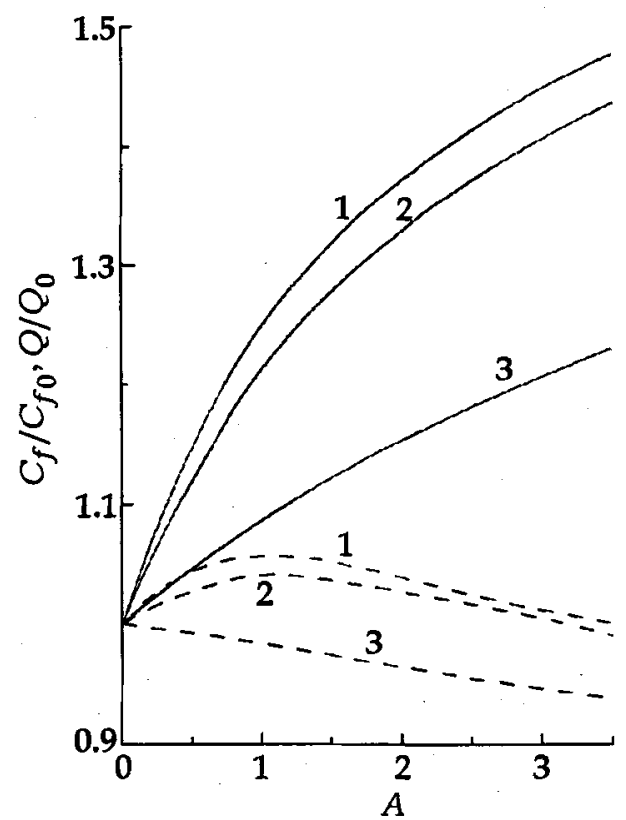

Fig.6 Ratios of the heat fluxes (solid) and friction coefficients (dashed) in dusty and pure gases at the stagnation point of an axisymmetric blunt body in hypersonic flow. $\beta_{0}=8.01,8.1,12$ (curves $1 \sim 3), c_{p} / c_{s}=1$ particles, which is sufficiently high in the boundary layer. The calculations demonstrated that the greater $c_{s} / c_{p}$, the greater the effect of the particles on the heat flux. The variation of $T_{w}$ over the range $0 \leq T_{w} \leq 0.99$ has almost no influence on the results presented in Fig. 6 . The calculations demonstrated also that the consideration of the boundary layer eliminates the particle concentration singularity on the wall. 
The calculation results of the nonstationary boundary layer are presented in Fig.7 for $\beta_{0}=8.1, c_{s} / c_{p}=1$. Curves 1,2 correspond to the second (step-shaped) type of the concentration nonuniformity. Clearly, the relative heat flux and friction coefficient vary monotonously from the steady-state values corresponding to $n_{s f}=1$ to the values corresponding to $n_{s f}=2$, therewith the width of the transition region depends on $\Omega$ only slightly. Curves $3 \sim 5$ correspond to the harmonic nonuniformities of the free-stream particle concentration with $\Omega=0.2,0.5,1$, respectively. Clearly, with decrease in the nonuniformity period the amplitude of the pulsation of both the heat flux and the friction coefficient also decreases and the heat flux and friction coefficient tend to the corresponding values for a steady-state flow with its constant free-stream particle concentration equal to the mean concentration in the nonuniformity. As the nonuniformity period increases, the heat flux and the friction coefficient tend to the corresponding quasi-steadystate values and hence the most "dangerous" nonuniformities (resulting in the largest amplitudes of the heat flux pulsations) are the nonuniformities with $\Omega<0.1$.

\section{SUMMARY}

Numerical modeling of the nonstationary effects in the shock and boundary layers on a blunt body traveling at hypersonic speed in nonuniform dusty gas with small particles demonstrated that the gradients of the particle concentration nonuniformity sharply increase with decrease in the distance from the body surface. For step-shaped nonuniformities of the particle concentration, the nonstationary heat flux and the friction coefficient at the stagnation point vary monotonously between the initial and finite steady-state values corresponding to the initial and finite free-stream particle concentrations. For harmonic nonuniformities, it is found that as the nonuniformity period decreases the heat flux at the stagnation point tends to the steady-state value for uniform free-stream flow with the mean particle concentration. With increase in the nonuniformity period (beginning with $\Omega=0.1$ ), the heat flux tends to the corresponding quasi-steady-state value.

\section{REFERENCES}

1 Humphrey JAC. Fluid motion in erosion by solid particle impact. Int $J$ Heat and Fluid Flow, 1990, 11(3): 170 195

2 Fleener WA, Watson RH. Convective heating in dust laden hypersonic flows. AIAA Paper No 
73-761, 1973

3 Dunbar LE, Courtney JF, McMillen LD. Heating augmentation in erosive hypersonic environments. AIAA J, 1975, 13: 908 912

4 Shih WCL. Ballistic range measurements of aerodynamic heating in erosion environments. AIAA Paper No 76-319, 1976

$5 \mathrm{Yu} \mathrm{HR}, \mathrm{Li} \mathrm{ZF}$, Lin JM. Shock tube measurements of stagnation point heat transfer rates in dusty flow. In: Proc 16th Int Symp Shock Tubes and Waves, Aachen, West Germany, July 26 31, 1987. 243 249

6 Mikhatulin DS, Polezhaev Yu V, Repin IV. Heterogeneous flows: gasdynamics, heat transfer, erosion. Preprint Inst High Temp RAS, 1987, (2-402): 87 (in Russian)

7 Osiptsov AN. Boundary layer on a blunt body in dusty gas flow. Izv Akad Nauk SSSR, Mekh Zhidk Gaza, 1985, (5): 99 107

8 Osiptsov AN, Shapiro EG. Effect of a dispersed admixture on the structure of the boundary layer on a blunt body in hypersonic flow. Izv Akad Nauk SSSR, Mekh Zhidk Gaza, 1986, (5): $55 \sim 62$

9 Osiptsov AN, Vasilevskii EB. Heat transfer in a stagnation region of a blunt body in a hypersonic gas flow with an admixture of tiny particles. Theory and first experimental results. In: Proc 2nd Int Conf Two-Phase Flow Modelling and Experiment. Pisa, Italy, May 23 26, 1999, 75 82

10 Marble FE. Dynamics of dusty gases. Ann Rev Fluid Mech, 1970, 2: 397 446

11 Carlson DJ, Hoglund RF. Particle drag and heat transfer in rocket nozzles. AIAA J, 1964, (2): 1980 1984

12 Hayes WD, Probstein RF. Hypersonic Flow Theory. New York: Acad Press, 1959

13 Lunev VV. Hypersonic Aerodynamics, Moscow: Mashinostroenie, 1975 (in Russian)

14 Osiptsov AN, Rozin AV, Teverovskii MA, Wang BY. Novel Lagrangian method for calculating the particle concentration in dusty-gas flows with intersecting particle trajectories. In: Proc 3d Int Conf Multiphase, Non-Newton, Phys-Chem Flows, Beijing, China, October 7 10, 1997. 2-65 2-72

15 Osiptsov AN, Shapiro EG. Dusty-gas flow past a sphere at a high supersonic velocity. In: Investigation of Gasdynamics and Heat Transfer in Complex Flows of Homogeneous and Multiphase Media. Moscow: Moscow State Univ, 1990. 89 105 (in Russian)

16 Osiptsov AN. Investigation of regions of unbounded growth of particle concentration in dispersed flows. Izv Akad Nauk SSSR, Mekh Zhidk Gaza, 1984, (3): 46 52

17 Cole JD. Perturbation Methods in Applied Mathematics. Blaisdell, Waltham, MA. 1972

18 Osiptsov AN. Mathematical modeling of dusty-gas boundary layers. Appl Mech Rev, 1997, 50(6): $357 \sim 370$ 